\title{
MEASURING THE PERFORMANCE OF LEVERAGED AND NON-LEVERAGED ETF'S
}

\author{
Martin Širůček ${ }^{1}$, Václav Ruml², Petr Strejček ${ }^{1}$ \\ ${ }^{1}$ Department of Finance, Faculty for Business and Economics, Mendel University in Brno, Zemědělská 1, 61300 \\ Brno, Czech Republic \\ ${ }^{2}$ KPMG Czech Republic, Veveří 3163/111, 61600 Brno, Czech Republic
}

To cite this article: ŠIRU゚ČEK MARTIN, RUML VÁCLAV, STREJČEK PETR. 2018. Measuring the Performance of Leveraged and Non-Leveraged ETF's. Acta Universitatis Agriculturae et Silviculturae Mendelianae Brunensis, 66(5): 1357-1367.

To link to this article: https://doi.org/10.11118/actaun201866051357

\begin{abstract}
This paper deals with exchange traded funds (ETFs) and valuation it 's performance according to selected indicators. For empirical analysis 10 leveraged and non-leveraged ETFs listed on US market is chosen according to selected criterias (adequate history at least 7 years, daily presented NAV, accessibility for retail investor). Observed time period was 2010-2015 and selected investment horizon is 1, 3 and 6 years. Funds are analyzed on the basis of NAV in the terms of return and risk represented by selected indicators (like Sharpe ratio, Traynor ratio, Information ratio, Apparaisal ratio and indicators like alfa (Jensen Alfa) and beta. Results are commented in a broader context in summary and discussion chapter as well as recommendations. Measured by classical Sharpe ratio, both groups bring to investor pretty same results, but e.q. by Information ratio by non-leveraged ETF shows very clearly the importance of work by ETF portfolio manager. Only a few leveraged ETF bring to the investor adequate ratio between profit and level of risk.
\end{abstract}

Keywords: ETF, systematic risk, tracking error, standard deviation, specific risk

\section{INTRODUCTION}

Investing in exchange-traded funds (ETFs) is a well-known and frequently employed method of investments or speculations. ETFs are a flexible and fast growing group of funds, which give rise to a number of specific kinds. As stated by Hill, Nadig and Hougan (2016), in early 2015 the assets of exchange-traded funds and notes globally were 2.9 trillion USD according to ETFGI ${ }^{1}$, with 2 tril. USD in the United States. That amount represents over 5,000 individual ETFs from 247 providers listed on 63 exchanges in 51 different counters. One of the often discussed and, as needs to be noted, criticised funds are leveraged ETFs.

The presented paper is focused on comparing classic ETFs with the later and more risky funds, i.e. leveraged ETFs. These funds are compared in terms of performance analysis using selected indicators, which will be specified in this methodology. Attention is also paid to the fact whether the leveraged ETFs are more suitable for short-term type of investment or speculation or whether they should be kept for longer periods of time. Cheng, Madhavan (2009) and Little (2010) argue that leveraged ETFs do not deliver the returns investors

1 ETFGI LLP Press Release from 9 April 2015. ETFGI LLP is an equity research firm. It provides proprietary research on the global exchange traded fund and exchange traded products industry. 
may expect when they invest in them for periods longer than a day or two. On the other hand, Guedj, $\mathrm{Li}$, Mcann (2010) find that many investors hold their leveraged ETFs for very long periods, longer than three months. With respect to ETF investment or speculation it is also interesting to observe how the returns and risk develop over time in relation to the specific types of funds.

The objective of this paper is to define recommendations for the investor/speculator based on the analyses of leveraged and non-leveraged ETFs with respect to the returns and risk of the selected ETFs and the investment horizon duration.

\section{Literature review}

According to Meziani (2006), one of the major differences, distinguishing ETFs from standard mutual funds is that the owner of this type of fund issues securities of the ETF fund with the trading being continuous depending on the market. Typically, the ETF price is affected by the relevant demand and supply and the issuer is therefore not obliged to determine purchase and selling prices as is the case of investment certificates.

An important feature of ETFs is their variety. There are funds specializing in specific indices, commodity or currency as well as funds whose underlying assets cover all of the above areas. The popularity of ETF is demonstrated by Factbook (2016) as well as intraday tradability, price transparency, tax efficiency and access to specific markets or asset classes.

Most often, the ETFs are divided according to the type of underlying assets. An alternative may be distribution according to the objective that the fund seeks to fulfil. Examples of these are index ETFs that are a typical example of the type of fund trying to copy a particular benchmark. Similarly, there are funds seeking to perform in a manner copying certain sectors, commodities or currencies. Contrariwise, there are funds that aim to overcome such predetermined benchmarks, i.e. actively managed funds. According to Burns (2009), the first actively managed ETFs in the US market (and globally) was Grail American Beacon Large Cap Value ETF established by Grail Advisor in early 2009. As stated by Shum (2011), over the last few years, the market for Exchange-Traded Funds (ETFs) has grown exponentially, there were more than 1200 ETFs listed in the U.S. and Canada in the spring of 2010.

The most marketable type of ETFs is Broad-based ETF where these ETFs rank amongst the top 100 marketable ETFs in the American market ${ }^{2}$. As stated by Hassine, Roncalli (2013), for passive managers, absolute performance is meaningless. In an ideal world, they would like to buy and sell the investment at any moment and have exactly the same return as the index. Passive managers thus focus on factors other than absolute return: management fees and other costs, tracking error volatility, liquidity, structuration risks.

While the above types of ETFs are standard (non-leveraged) types of funds, it is now essential to focus on the main representative of the specific type of ETFs. In addition to other specific types such as dividend or currency ETFs, leveraged ETFs are now subject to a considerable increase in interest. As stated by Shum (2011), the management of leveraged ETFs differs significantly from that of regular ETFs. These funds aim to track daily returns, instead of the price of an underlying index at a higher frequency (e.g., 15-minute intervals). Leverage and derivative securities such as total return swaps and future contracts are employed by the leveraged ETF manager (or a third-party structured product specialist) to magnify the underlying index's return or its inverse by two or three times. The portfolio is rebalanced once a day at the end of the trading day. The leveraged ETF's end-of-day net asset value (NAV) is made known to authorised participants, and so an arbitrage mechanism - albeit in cash rather than in kind - also exists to help keep the market price of the leveraged ETF close to its NAV, at least at the end of the trading day. The increasing popularity of leveraged ETFs is explained by Cheng, Madhav (2009) through three factors. First, these funds offer short-term traders and hedge funds a structured product to express their directional views regarding a wide variety of equity indices and sectors. Second, as investors can obtain levered exposure within the product, they need not rely on increasingly scarce outside capital or the use of derivatives, swaps, options, futures, or trading on margin. Third, individual investors - attracted by convenience and limited liability nature of these products - increasingly use them to place longer-term leveraged bets or to hedge their portfolios.

Although the market has had experience with leveraged funds since the 1990s, leveraged ETFs only came into existence in the second decade of the new millennium. Specifically, in the middle of 2006 when the first leveraged ETF, Pro Shares UltraS \& P 500 (SSO), started being traded. As stated by Cheng, Madhav (2009), the category has exploded since the first products were introduced in 2006, especially in volatile sectors such as Financials, Real Estate, and Energy. There are now over 106 leveraged and inverse ETFs in the US with Assets Under Management (AUM) worth about 22 bil. USD.

The expense ratio is used to determine the fund costs. In simplified terms, this indicator, which is updated on a daily basis, is determined as the value 
of all the costs of the relevant fund for one year divided by the value of assets managed by the fund. In practice, however, the expense ratio is not so easy to determine, and multiple variables must be taken into account. The investor must consider a whole range of expenses related to establishment and management of the fund. One of the examples is the management fee (sometimes referred to as the advisory fee) charged by the fund manager such as Vanguard and BlackRock for their advisory and investment services. Other expenses, referred to as administrative expenses, include legal services, accounting and the actual fee for listing at the New York Stock Exchange (Nadig, 2014). On other hand the expense ratio does not include other expenses, such as transaction fees. Such a commission fee most often totals units or a few tens of dollars at most, depending on the provider.

Besides the aforementioned operation expenses, the investor encounters another type of fees, i.e. bid-ask spreads. ETF managers seek to ensure that the value of their funds is as close as possible to the net asset value. A problem may arise in situations with low volume ETFs where there is often a wide spread. Trading a broad-spread fund is a hidden cost to the investor as this spread affects the selling, i.e. purchase price, thereby reducing the potential revenue for the investors (Pylypczak-Wasylyszyn, 2015).

\section{MATERIALS AND METHODS}

To achieve the objective set by this study, a representative sample (division according to underlying assets, sectors, etc.) of leveraged (10) and non-leveraged (10) ETFs tradable in the American market was selected, meeting the following criteria: (a) adequate fund history (the fund foundation date must be before 2010), (b) fund accessibility to standard investors, (c) access to daily historic NAV values.

The benchmark is always the S \& P 500 index. The recommended time horizon for share funds' investments is 5-10 years. Given the fact that the first leveraged ETF was established as late as 2006 and given the ten-year interval, there is lack of data to be entered into the analysis. For this reason, the period selected for the analysis runs for 1,3 and 6 years, which means the period from 1. 1.2010 to 31. 1.2015. Given the duration of the shortest selected period (1 year), we selected a 14-day time period to acquire a sufficient data set.

As the risk free rate, we selected the $3 \mathrm{M}$ Treasury Bill Rate return. The individual ETFs will be compared in the analysis using the calculated returns and risk, returns above the risk free rate and Sharp ratio.

According to Fabozzi, Markowitz (2011), the ETF return is calculated using the average returns, i.e. annualised returns based on geometric mean.

$$
r_{\text {annaul }}=\left[\prod_{i=1}^{n}\left(1+r_{i}\right)\right]^{\frac{m}{n}}-1
$$

Where $m$ stands for a one-year time horizon (365 for daily data, 52 weekly data, etc.) and $n$ is the number of periods that are relevant for a given return.

Various variability rates are used as the basic methods serving as a risk indicator. Such methods include, for example, dispersion or average absolute variance. One of the best known and most widely used methods for calculating the risk is the standard deviation of returns (2). It consists of the above-mentioned dispersion and is defined as follows:

$\sigma=\sqrt{\frac{1}{n-1} \sum_{i^{\prime} 1}^{n}\left(r_{i}-\bar{r}\right)^{2}}$

According to Solnik, McLeavy (2004), it is also necessary to take into account for what period we seek to obtain the values and in what intervals these periods are repeated. For these purposes it is necessary to use the so-called annualised standard deviation where the standard deviation of returns is multiplied by the so-called annualization coefficient ( $\sqrt{t}$, i.e. $\sqrt{12}$ for monthly data, $\sqrt{4}$ for quarterly data, $\sqrt{52}$ for weekly data, $\sqrt{251}$ for daily data, etc.).

The investment risk is also linked to the Sharpe ratio indicator defined by Litterman (2004) as the difference between total returns and risk free returns (i.e. returns above the risk free rate of return) relative to the investment risk rate (standard deviation).

$$
S R=\frac{r-r_{F}}{\sigma}
$$

In the formula (3) $r$ is the portfolio return, $r_{F}$ stands for risk free returns (T-bill return) and the standard deviation divides all values in the denominator. Standard deviation is the measurement of total risk. Standard deviation contains so systematic (market) risk and unsystematic risk.

In order to monitor the portfolio performance it is advisable to compare it with the selected benchmark, which ETF seeks to copy. The regression analysis is based on determining the benchmark and the portfolio return values using regression functions. The basic form of the regression equation then consists of several parameters, of which two coefficients are crucial for further methods, i.e. alpha and beta coefficients. According to Fabozzi, Markowitz (2011), the alpha coefficient indicates with respect to regression analysis the distance from the regression line and the beta coefficient indicates the slope of this line. By expanding the regression, equation by risk-free rate of return the CAPM model can be obtained. In mathematical terms, it is the defined as follows:

$r-r_{F}=\alpha+\beta \cdot\left(b-r_{F}\right)+\varepsilon$ 
where $b$ is the return on benchmark (market profit) and $\varepsilon$ stands for the value residue from the regression line. Beta coefficient $(\beta)$ represent the systematic risk.

The previously formula (4) is then used to determine beta following the mathematical expression below:

$$
\beta=\frac{\sum_{i=1}^{n}\left[\left(r_{i}-r_{F}-\bar{r}-\overline{r_{F}}\right) \cdot\left(b_{i}-r_{F}-\bar{b}-\overline{r_{f}}\right)\right]}{\sum_{i=1}^{n}\left(b_{i}-r_{F}-\bar{b}-\overline{r_{F}}\right)^{2}}
$$

Beta coefficient in formula (5) expresses the rate of risks of funds relative to the benchmark (the whole market). In essence, it expresses the rate of systematic risk, i.e. The risk that affects everyone equally and cannot be directly influenced. Moreover, as the systematic risk cannot be diversified, and as beta coefficient is its indicator, the systematic risk can be calculated as the product of beta and the benchmark standard deviation of returns. (Fabozzi, Markowitz, 2011).

Szylar (2010) states that beta coefficient can be divided into beta + , resp. beta-. This is the calculation of beta coefficient for growing $($ beta + ) and declining (beta - ) markets. In the case of a growing market, it is desirable that beta be higher than 1 as the value of portfolio overruns this benchmark value. In the case of bear market, it is on the other hand better if beta coefficient is below 1, i.e. The fund value drops less than the market value (benchmark).

The second parameter of the regression equation is alpha coefficient, i.e. Jensen's alpha, which Jensen (1967) defines as a value of excess return reduced by the systematic risk. Therefore, it can be determined that this is an additional extra yield compared to what would correspond to the undertaken risk.

$\alpha=r_{P}-r_{F}-\beta \cdot\left(b-r_{F}\right)$

Another indicator is the Treynor ratio. According to Solnik, McLeavy (2004), this indicator compares the return above the risk free rate to the systematic risk (not to the entire risk of investment; therefore, it does not take into account non-systematic risk).

$T R=\frac{r-r_{F}}{\beta}$

The link between the Sharp ratio and Jensen's alpha gives rise to another indicator, Appraisal ratio, which, according to Aragon, Ferson (2006) focuses on specific risk $\left(\sigma_{\varepsilon}\right)$, unlike Jensen's alpha. The excess return is cleared of the systematic risk (Jensen's alpha) in the numerator and divided by the non-systematic risk. Thus, this indicator measures the quality of selecting assets in the portfolio compared to the risk they represent.
$A R=\frac{\alpha}{\sigma_{\varepsilon}}$

The study also uses relative risk indicators, specifically the Tracking error and Information ratio. Fabozzi, Markowitz (2011) define the tracking error as the deviation of the returns relative to the benchmark. Thus, this is the standard deviation of fund returns relative to the benchmark returns.

The Tracking error of the active portfolio $x$ with respect to the benchmark is the difference between the return of the portfolio $R(x)$ and the return of the benchmark $R(b)$ :

$$
\begin{aligned}
& e=R(x)-R(b)=\sum_{i=1}^{n} x_{i} R_{i}-\sum_{i=1}^{n} b_{i} R_{i}= \\
& =x^{\mathrm{T}} R-b^{\mathrm{T}} R=(x-b)^{\mathrm{T}} R
\end{aligned}
$$

As mentioned by Hasine, Roncalli (2013), the objective of the investor is to maximise the expected Tracking error with a constraint on the tracking error volatility:

$x^{*}=\arg \max (x-b)^{\mathrm{T}} \cdot \mu$

Grinold, Kahn (2000) or Hasinne, Roncali (2013) recommend comparing the performance of different portfolios, a better measure than the Sharpe ratio is the Information ratio, which is defined as follows:

$\operatorname{IR}(x \mid b)=\frac{\mu(x \mid b)}{\sigma(x \mid b)}=\frac{(x-b)^{\mathrm{T}} \cdot \mu}{\sqrt{(x-b)^{\mathrm{T}} \cdot \sum(x-b)}}$

Solnik, McLeavy (2004) define the information ratio as an excess return over the benchmark. Tůma (2014) defines this indicator more specifically as active returns to active risk.

\section{Data description}

To compare classic and leveraged ETFs, a total of 10 representatives were selected from each area. The choice fell not only on ETFs that are validated and strong in the market given the size of managed assets but also on various ETFs from areas such as the financial sector or energy. The selected market is the American market, which offers 1.594 ETFs and 2.1 trillion USD in assets under management according to ICI Factbook (2016). Tab. 1 shows the selected non-leveraged and leveraged ETFs that enter the empirical analysis.

\section{RESULTS}

In terms of expenses, leveraged ETFs are by far a more expensive instrument in the market. Values related to this type of ETF range from 0.9 to $1 \%$. The most expensive "classic" ETF is iShares U.S. Financials ETF reaching over four tenths of percent of the expense ratio. 
I: Assets values of selected ETF's

\begin{tabular}{cccc}
\hline Leveraged ETF 's & $\begin{array}{c}\text { AUM } \\
\text { (mld. USD) }\end{array}$ & Non-leveraged ETF's & $\begin{array}{c}\text { AUM } \\
\text { (mld. USD) }\end{array}$ \\
\hline Pro `Shares Ultra S \& P 500 & 1.46 & SPDR S \& P 500 ETF Trust & 197.22 \\
\hline Direxion Daily Financial Bull 3× Shares & 1.03 & iShares Core S \& P 500 ETF & 79.87 \\
\hline ProShares Ultra QQQ & 0.86 & Vanguard Total Stock Market Index Fund & 468.74 \\
Direxion Daily Small Cap Bull 3× Shares & 0.68 & Vanguard Value Index Fund & 44.49 \\
ProShares Ultra Financials & 0.66 & iShares Core S \& P 500 Small Cap ETF & 20.61 \\
Pro Shares Ultra Pro S \& P500 & 0.65 & Vanguard Total Bond Market Index Fund & 174.85 \\
Direxion Daily Energy Bull 3× Shares & 0.45 & Energy Select Sector SPDR Fund & 14.74 \\
Direxion Daily S \& P 500 Bull 3× Shares & 0.46 & iShares U.S. Financials ETF & 1.55 \\
\hline Pro Shares Ultra Real Estate & 0.18 & SPDR S \& P BIOTECH ETF & 2.32 \\
\hline Direxion Daily Technology Bull 3× Shares & 0.17 & Vanguard REIT Index Fund & 60.13 \\
\hline
\end{tabular}
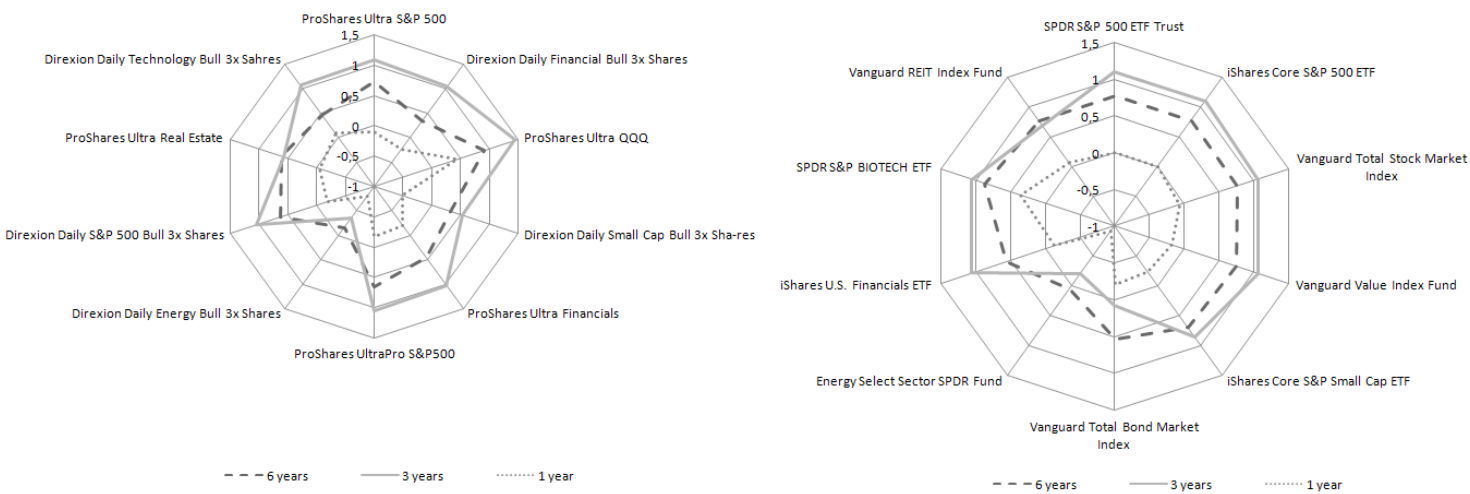

1: Sharpe ratio leveraged and non-leveraged ETFs
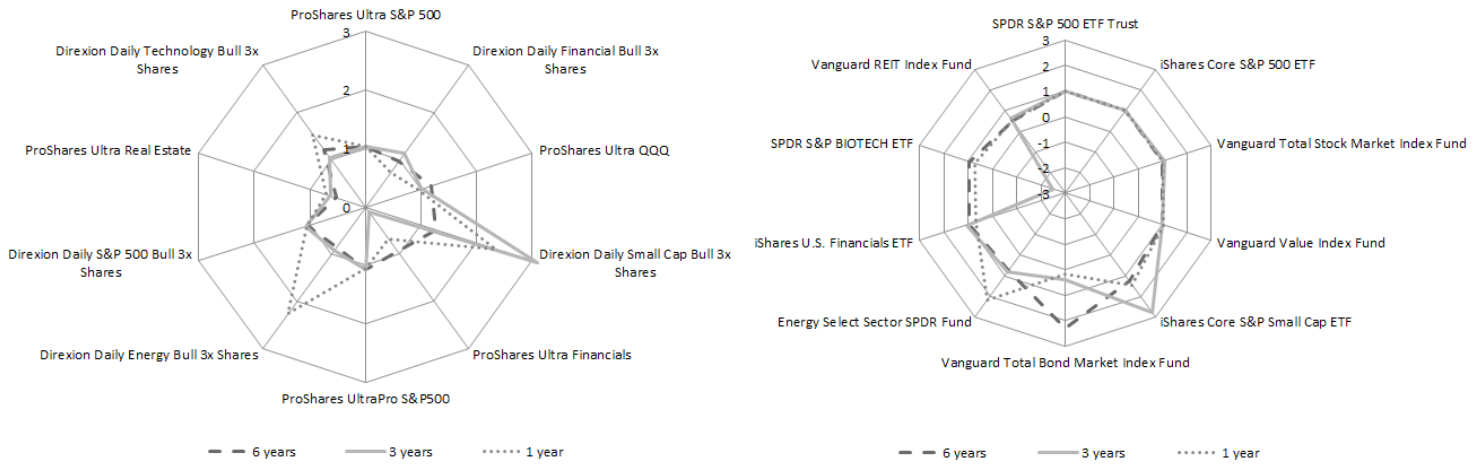

2: Timing ratio leveraged and non-leveraged ETFS

The Fig. 1 shows the Sharpe ratio of selected leveraged and non-leveraged ETFs. Here the difference between the classic and leveraged ETFs is not so obvious, perhaps except for the longest 6-year period under review. While the values for the two of the longest periods of time are generally positive, the opposite is the case with respect to the shortest period. Thus, the real returns were largely negative with a few exceptions. The highest is again the leveraged ProShares Ultra QQQ. For this ETF the indicator defines that each percent of the standard deviation of returns yields $0.44 \%$ of returns for the fund (p.a.).
The average standard deviation (standard risk) of revenues (see Appendix 1) of leveraged ETFs is nearly $40 \%$, whereas for classic ETFs it is only $15 \%$. What is interesting is that the most profitable classic ETFs compare favourably with leveraged funds in terms of variability and some even overcome them. Again, this confirms the equation that the higher the returns on the investment, the higher the risk to be taken. The least risky ETFs are those focusing on bonds.

Furthermore, indicators based on regression analysis built upon the CAPM model have been analysed. Risks (beta coefficients) for each ETF have been calculated, including bull beta (beta + ) and 
bear beta (beta -), and the Timing ratio indicator. This analysis has confirmed that ETF with triple leverage actually achieves a beta coefficient higher than three. With respect to positive beta it is interesting to note that $70 \%$ of leveraged ETFs react to the bull market better than the benchmark, while with classic ETFs only one third is so successful (see Appendix 2). However, if the market is declining, a significantly better protection of classic ETFs against market slumps can be observed. In a declining market, the returns are lost on a lower scale than the benchmark by about half of the leveraged analysed ETFs.

The timing ratio indicator (see Fig. 2) defines how well the fund managers react to market fluctuations. What is worth mentioning are leveraged ETF Direxion Daily Small Cap Bull 3× Shares, which manage to react to the bull as well bear markets very well (the value of 3.09 in 3-year investment horizon).

In the next part of the analysis, two types of risks have been considered - systematic risk (beta coefficient) and specific risk. The systematic (non-diversified) risk ranges from 15.5-11.5\%, for the relevant classic ETFs, which corresponds to standard values. Logically, the leveraged ETFs are riskier, as confirmed by the analysis showing that the risk rate usually increases with the duration of the investment horizon. On average, leveraged ETFs show three times higher risk rate compared to the classic ones with respect to the systematic risk, which corresponds to their triple leverage. As regards the specific risk, the risk of leveraged ETFs was on average only "double" compared to non-leveraged ETFs. It has therefore been confirmed that even with leveraged instruments the risk can be reduced using appropriate diversification. Thanks to the suitable choice of assets and portfolio diversification, the non-leveraged ETFs SPDR S \& P 500 ETF Trust or iShares CoreS \& P 500 ETF show low specific risk rates. Leveraged ETFs have the lowest risk rate thanks to the appropriate diversification (broad diversification to S \& P 500 index) of underlying assets such as Pro Shares UltraS \& P 500 and Direxion Daily S \& P 500 Bull 3× Shares. The values of the specific and systematic risk over individual investment horizons are presented in Appendix 3. The risk analysis included regression analysis, i.e. determination Index $\left(\mathrm{R}^{2}\right)$ analysis, which defines what revenues/loss rate can be explained by the benchmark movement. The closer $\mathrm{R}^{2}$ approaches 1, the greater the share of systematic risk in the overall investment risk. Conversely, the closer $\mathrm{R}^{2}$ approaches 0 , the more the ETF volatility is caused by factors other than market (benchmark) volatility (movement). Based on the regression analysis results, the threshold value for considering ETF for further analysis has been selected with respect to Nau (2016) as the critical value of 0.7. Of the original 10 non-leveraged ETFs,
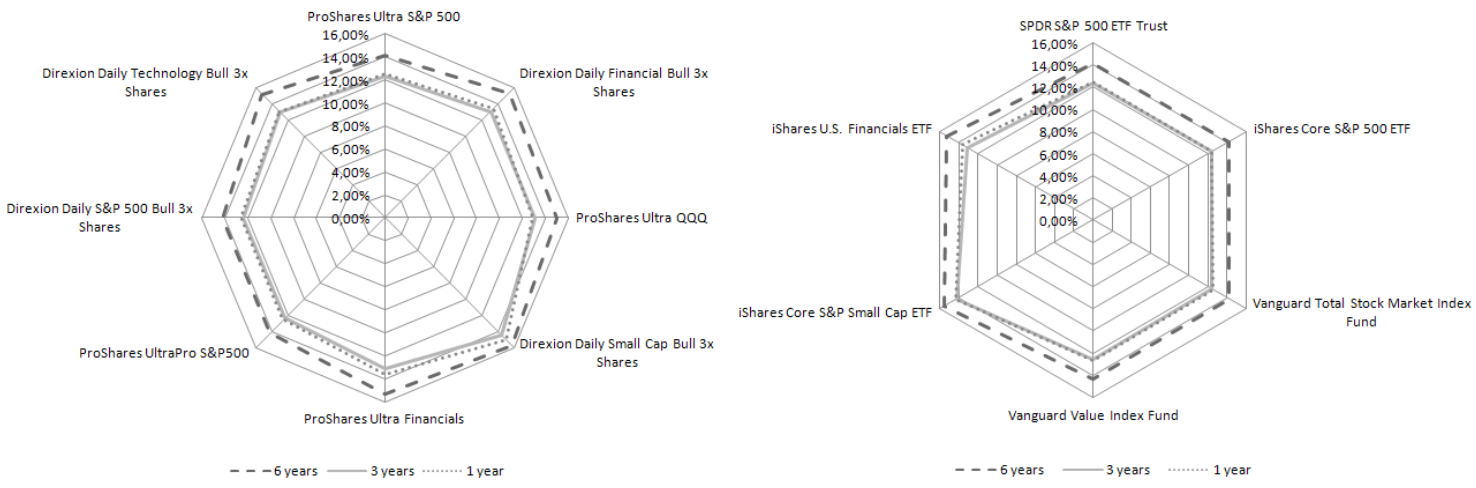

3: Traynor ratio leveraged and non-leveraged ETF's
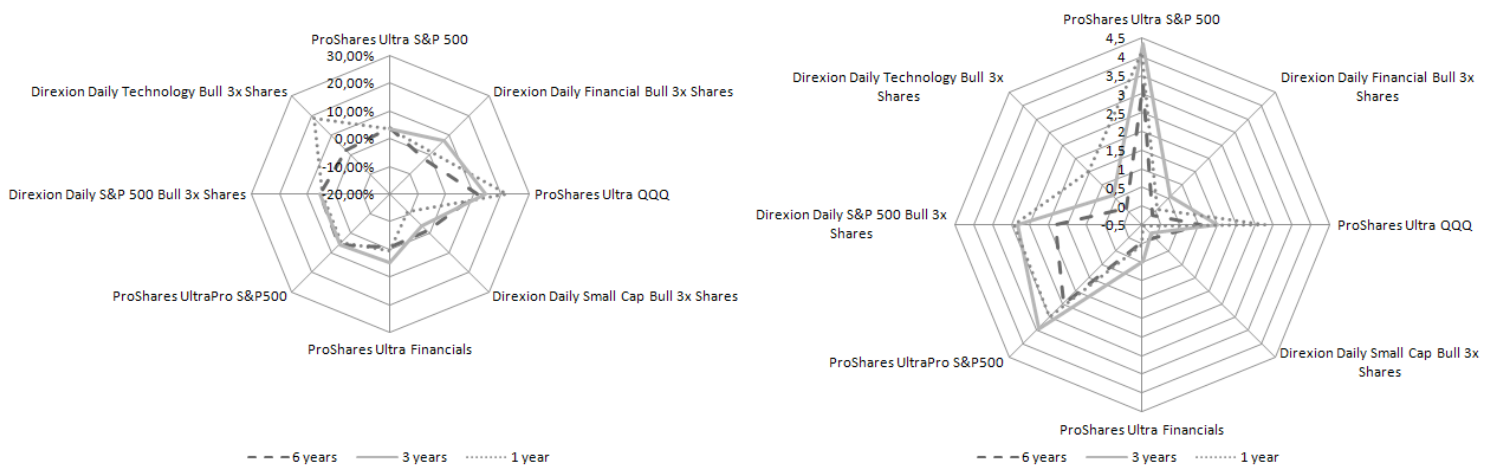

4: Jensen's alpha and Appraisal ratio leveraged ETFs 


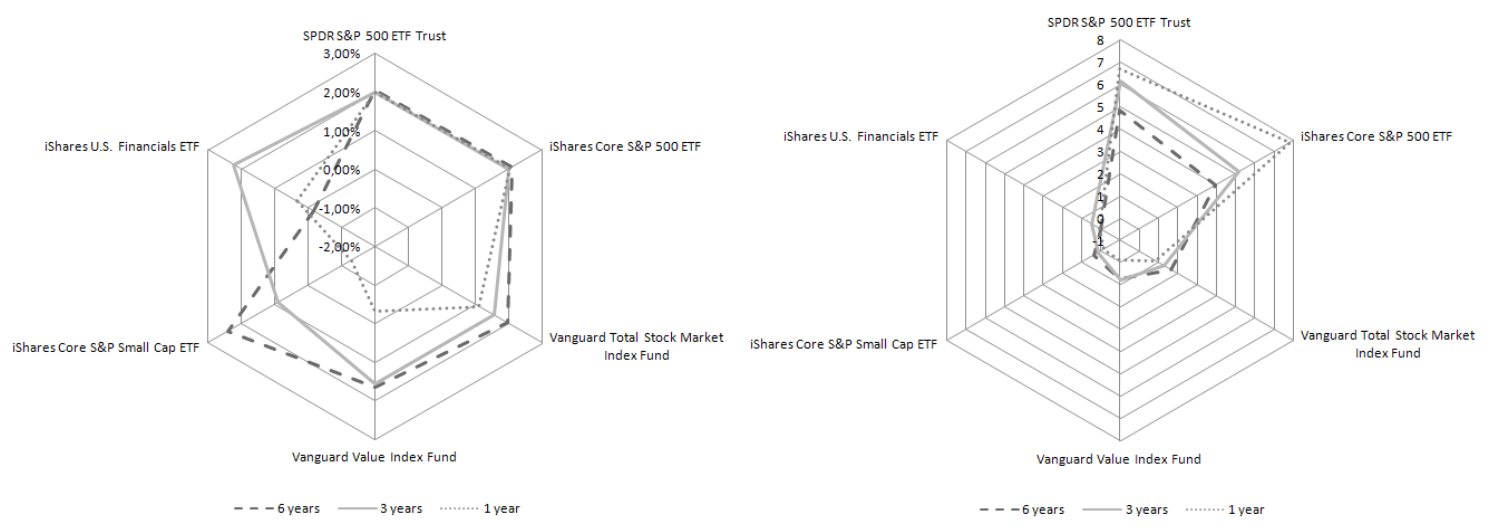

5: Jensen's alpha and Appraisal ratio, non-leveraged ETFs
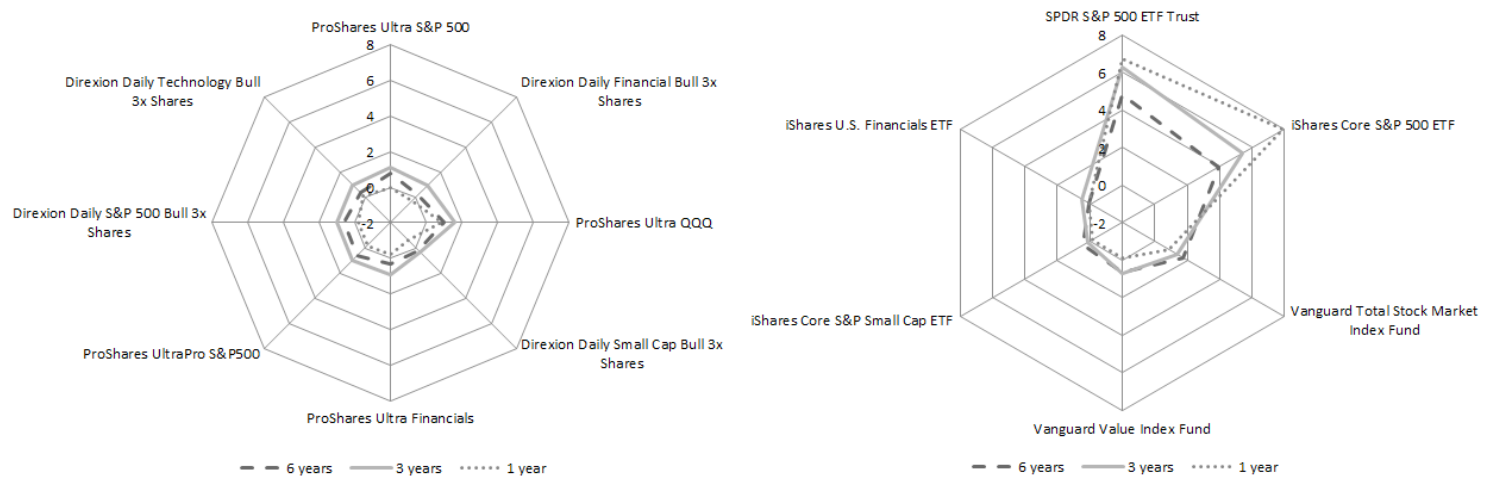

6: Information ratio of leveraged and non-leveraged ETFs

only 6 ETFs with $\mathrm{R}^{2}$ higher than 0.7 have thus been included in the next analyses.

The next part of the analysis has been dedicated to selected investment indicators such as Treynor ratio, Jensen's alpha and Appraisal ratio, which are based on the regression analysis. In addition, attention has been paid to the deviation from benchmark (Tracking Error) and the ability to make use of this deviation (Information ratio).

From the point of view of leveraged ETFs, the fund focusing on small-cap companies has ranked best in all the observed periods. It has also won over its "competitor" iShares Core S \& P Small ETF - a classic ETF (see Fig. 3). ETFs with comparable specifics risks also have comparable Treynor ratios, as more in-depth analyses show. This indicates that these ETFs are similarly strongly diversified.

As regards leveraged ETFs, the results are enhanced by individual leverages of the specific funds. The only fund which failed to beat the benchmark in any of the monitored period, is Direxion Daily Small Cap Bull $3 \times$ Shares. The reasons for the slump in this ETF, which can be observed mainly in the shortest periods, are not really related to the consequence of the risk (expressed by beta), but they are mainly related to the poor results in terms of the generated returns. In general, ProShares Ultra QQQ beats the benchmark in all periods by almost $21 \%$.
A similar result was achieved by the ETF with triple leverage Direxion Daily Technology Bull 3× Shares. In general, leveraged ETFs are able to overcome the benchmark three or four times more than the non-leveraged ETFs.

How Jensen's alpha indicator may become somewhat distorted can be seen in the example of the leveraged Direxion Daily Technology Bull $3 \times$ Shares ETF. In the shortest period of time, the alpha values of the fund were less than $18.5 \%$, and thus it was by far one of the two best. However, due to the high individual risk (12.25\%), its position considerably deteriorated in terms of the Appraisal ratio. Jensen's alpha and Apparisal ratio by leveraged ETF describe Fig. 4.

Classic ETFs do not reach the leveraged ETFs values (see Fig. 5). The highest alpha was recorded by iShares Core S \& P Small Cap ETF over a six-year period at 2.4\%. However, this type of ETF shows small result dispersion. What is worth mentioning is the small difference in alpha values in the two largest share ETFs in the market. Thus, both these funds overcome the benchmark steadily in all periods, which is certainly an interesting finding for each investor. However, in terms of the ability to beat the market, non-leveraged ETFs show worse results than leveraged ETFs.

While Jensen's alpha itself is focused on systematic risk, the Appraisal ratio indicator also considers 
the specific risk. In the short run, the best results are reported by two largest classic ETFs, followed by the largest leveraged ETF by ProShares Ultra S \& P 500. Also the next one ranks amongst leveraged ETFs which suggests that the managers of these funds make effective use of the specific risk.

While all FTEs beat the S and P 500 index over the six-year as well as the three-year period, the leveraged ETF succeed only exceptionally is the shortest periods. With respect to classic ETFs, the success rate is higher with only two funds failing to beat the index. The year 2015 was quite unfavourable in terms of stock market developments over the past six years. Thanks to the active management of leveraged ETFs, Direxion Daily Technology Bull 3x Shares, and especially ProShares Ultra QQQ beating the benchmark by more than $15.5 \%$, high returns were achieved also this year.

The final analysis focused on whether deviations from the benchmark pay off to the leveraged or non-leveraged ETF portfolio managers. The standard deviation of ETF and benchmark returns has been analysed, i.e. The Tracking Error. Whether a certain deviation from the benchmark (Tracking Error) pays off to the portfolio manager is evaluated by the ratio of active returns to active risk (due to a deviation from the benchmark) in the form of the Information ratio. Results if the portfolio manager brings to investor an active profit compared by active risk (Traking error) shows Fig. 6.

As regards non-leveraged ETF, these clearly show better work of the portfolio manager, i.e. more favourable deviation from the benchmark. The best results are achieved by the widely diversified classics ETF, iShares Core S \& P 500 ETF, respectively SPDR
S \& P 500 ETF Trust, which provides investors with nearly eight times the excess return in the yearly horizon versus the risk expressed as a deviation from the benchmark.

With respect to leveraged ETFs, the situation is quite different, i.e. with the shortest annual investment horizon the leveraged ETFs reach predominantly negative Information ratio values while the value slightly rises with prolonging horizons. The situation is opposite with respect to non-leveraged ETFs.

\section{DISCUSSION}

The aim of this paper was to analyse the performance of classic and leveraged ETFs traded in the US market, both in terms of profitability and risk. In addition to the above mentioned and described principles related to financial leverage (exponential growth of volatility supported by results in the form of a multiplicatively higher standard deviation), it is furthermore evident from the point of view of the relevant leveraged ETFs compared to the selected benchmark that these ETFs are significantly more sensitive in bear markets, i.e. they react more easily to the market downturns. This fact could be verified by negative beta in the regression analysis (see Appendix 2). With the declining market, the leveraged ETFs dropped faster than the benchmark in about half of the cases, while the classic ETF dropped in less than a third of the cases. When the market rises, it can be seen that they reach multiple returns of the selected index. The only exception is ProShares UltraS \& P 500 , which reacts identically in both directions.

\section{CONCLUSION}

The finding that is certainly worth noting is that a major percentage of leveraged ETFs generated significant returns over the six-year and three-year time intervals. In a number of expert articles and publications their authors strongly advise against keeping leveraged ETFs for a longer period of time. There are several reasons for that: the repeatedly mentioned high volatility, higher expenses and daily price rebalancing. All these cons of the leveraged ETFs are obvious from the individual indicators. Even so, despite such a small sample, there are cases when a longer-term investment can be considered.

The greatest surprise is the results of ProShares Ultra QQQ, ETF with double leverage. Using the simple but popular Sharpe ratio, this fund shows that it is by far the best across all the analysed funds, in all the periods under review. In addition, this indicator takes into account one of the main drawbacks of leveraged ETFs - volatility (risk).

As part of the testing, it was necessary to verify whether the selected funds are relevant to the market representative - the benchmark. The crucial point was the calculation of the determination coefficient and also the determination of threshold values of acceptability of 0.7 . Whether the selected level is sufficient is up to each investor to assess. This matter was dealt with in detail by Nau (2016) who elaborates on this indicator using the standard deviation or the random walk hypothesis. Results of his research were taken into account when choosing the threshold value of the determination coefficient.

Following the exclusion of those ETFs, the results of which in other indicators were statistically less accurate, further analyses were conducted using indicators comparing returns and risks from different perspectives. The Treynor ratio was not neglected, and its results favoured mainly the leveraged ETF. This indicator ignores the systematic risk, which reduces its reporting value mainly in the case of leveraged ETFs .

When the funds undertake a certain risk it is desirable that they be rewarded in the form of the so-called "excess returns". The funds were analysed for Jensen's alpha and the Appraisal ratio (also known as the Treynor-Black ratio). Here, ProShares Ultra QQQ is highlighted again as it beats all other ETFs by 
a long mile. Due to the worse results of the fund specific risk, it ranks lower from the point of view of the Appraisal ratio but it still beats all other classic ETFs save for the two largest ones.

In the last phase, the ETFs are compared against the benchmark performance. The development in share markets was considerably favourable over the analysed period (except for 2015). The major success is also evident in leveraged ETFs, which has not been left unanswered by a number of investors and investment firms. Some of them have also begun to address the issue of longer-term investments in this ETF type. Domelen (2015) analysed the performance of leveraged ETF SSO and UPRO in the case of a hypothetical zero Tracking error vis-à-vis S \& P 500 since 1950. The results were very favourable. In addition to the considerable simplification of such a model, two important items of information need to be pinpointed. First, it is highly unlikely that the leveraged type of the ETF would reach a Tracking Error approaching zero. The second factor highly distortive of Domelen's analysis was the period from which the historic data was taken. Years after the crisis are based on a wave of optimism with corresponding results of the leveraged ETFs. Longer predictions based on these few years would be highly misleading when applied over longer periods of time. This is basically a problem of using historic data and its interpretation for other time periods and future developments predictions. Based on the most recently analysed indicator - information ratio, it is advisable to compare the results with other analyses comparing leveraged and classic ETFs. One of them is the comparison of leveraged SSO with the well-known ETF Vanguard 500 Index Fund (VOO) (GavekalCapital.com, 2015). The analysis was conducted between 2011-2014 and its results are not very different in the specific indicators from the results of our analysis. Authors of the study also highlight the Information Ratio, which turns out surprisingly better for leveraged ETFs. Leveraged SSO achieve a principally higher Tracking error than classic ETFs. In their article they reached a value of $11.6 \%$, which is a realistic value based on the analysis calculations. However, the reported Information Ratio (8.1) would mean that SSO beats the benchmark by almost $94 \%$, which is not realistic.

What we agree with is the conclusion reached by authors of the article. Leveraged ETFs are undoubtedly a risky instrument. However, recent years of market development have turned this specific instrument into an investing tool even for longer-term portfolio diversifications.

\section{REFERENCES}

ARAGON, G. and FERSON, W. 2006. Portfolio Performance Evaluation. Hanover: now Publishers Inc.

BURNS, S. 2009. Actively Managed ETFs Are (Finally) Here. Morningstar. [Online]. Available at: http:// beta.morningstar.com/articles/271218/actively-managed-etfs-are-finally-here.html [Accessed: 2018, September 15].

DOMELEN, D. 2015. Performance Of Zero-Tracking Error S\&P 500 Leveraged ETFs Since 1950. Seeking Alpha. [Online]. Available at: http://seekingalpha.com/instablog/30945655-dane-vandomelen/3802666-performance-of-zero-tracking-error-s-and-p-500-leveraged-etfs-since-1950 [Accessed: 2018, September 15].

FABOZZI, F., and MARKOWITZ, H. 2011. The theory and practice of investment management. NewJersey: John Wiley \& Sons.

GAVEKALCAPITAL. 2015. Is That Leveraged ETF Worth It? Comparing SSO and VOO. [Online]. Available at: http://blog.gavekalcapital.com/?p=159 [Accessed: 2018, September 15].

GUEDJ, I., LI, G., and MCCANN, C. 2010. Leveraged ETFs, holding periods and investment shortfalls. Securities Litigation and Consulting Group, 2010. [Online]. Available at: https://pdfs.semanticscholar.org/ bdff/91la3bdd5dbb3afdf8dflea3337bff00da6e.pdf [Accessed: 2018, September 15].

GRINOLD, R. C., and KAHN, R. N. 2000. Active Portfolio Management: A Quantitative Approach for Providing Superior Returns and Controlling Risk. $2^{\text {nd }}$ Edition. McGraw-Hill.

HASSINE, M. and RONCALLI, T. 2013. Measuring the performance of exchange traded funds. The Journal of Investing, 4: 57-85

HILL, J. M., NADIG, D. and HOUGAN, M. 2016. A Comprehensive Guide to Exchange - Traded Funds (ETFs). 1. issue. CFA Institute Research Foundation.

CHENG, M. and MADHAVAN, A. 2009. Dynamics of leveraged and inverse ETFs. Q-group. [Online]. Available at: http://www.q-group.org/wp-content/uploads/2014/01/Madhavan-LeverageETF.pdf [Accessed: 2018, September 15].

ICI FACTBOOK. 2016. Investment Company Institute Factbook 2016. Invesment Company Institute.

JENSEN, M. 1967. The performance of Mutual Funds in Period 1945-1964. Journal of Finance, 23(2):389-416. LITTERMAN, B. 2004. Modern investment management: an equilibrium approach. New Jersey: John Wiley \& Sons. LITTLE, P. K. 2010. Inverse and Leveraged ETFs: Not your Fathers ETFs. Journal of Index Investing, 1(1): 83-89. MEZIANI, A. 2006. Exchange traded funds as an investment option. New York: Palgrave Macmillan.

NADIG, D. 2014. ETFs made easy: expense ratios. ETF.COM. [Online]. Available at: http://www.etf.com/ sections/blog/23937-etfs-made-easy-expense-ratios.html?nopaging=1 [Accessed: 2018, September 15]. 
NAU, R. 2016. What's a good value for R-squared? Statistical forecasting: notes on regression and time series analysis. [Online]. Available at: https://people.duke.edu/ rnau/rsquared.htm [Accessed: 2018, September 15].

PYLYPCZAK-WASYLYSZYN, D. 2015. The Hidden Costs of ETF Investing. ETFdb.com. [Online]. Available at: http://etfdb.com/etf-education/the-hidden-costs-of-etf-investing/ [Accessed: 2018, September 15].

SOLNIK, B. and MCLEAVY, D. 2004. International Investments. Boston: Pear-son Addison Wesley.

SHUM, P. 2010. How passive are international ETFs? A study of their intraday behavioral. Journal of Index Investing, 1(3): 74-84.

SZYLAR, C. 2010. Risk Management under UCITS III/IV: new challanges for the fund industry. London: ISTE Ltd. TŮMA, A. 2014. Priovodce úspěšného investora: vše, co potřebujete védét o fondech. $1^{\text {st }}$ Edition. Praha: Grada Partners.

Contact information

Martin Širůček: martin.sirucek@mendelu.cz

Václav Ruml: vaclav.ruml@mednelu.cz

Petr Strejček: strejcek@mendelu.cz

\section{APPENDIX}

Appendix 1: Standard deviation of selected leveraged and non-leveraged ETF's

\begin{tabular}{|c|c|c|c|c|}
\hline & \multirow{2}{*}{ ETF } & \multicolumn{3}{|c|}{ Standard deviation } \\
\hline & & 6 years & 3 years & 1 year \\
\hline \multirow{10}{*}{ 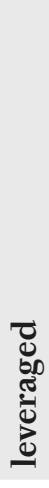 } & ProShares UltraS \& P 500 & $28.56 \%$ & $24.96 \%$ & $25.34 \%$ \\
\hline & Direxion Daily Financial Bull 3× Shares & $50.81 \%$ & $39.58 \%$ & $39.13 \%$ \\
\hline & ProShares Ultra QQQ & $32.92 \%$ & $27.87 \%$ & $29.94 \%$ \\
\hline & Direxion Daily Small Cap Bull 3× Sha-res & $55.38 \%$ & $46.57 \%$ & $44.01 \%$ \\
\hline & ProShares Ultra Financials & $33.68 \%$ & $26.13 \%$ & $25.87 \%$ \\
\hline & ProShares Ultra Pro S \& P500 & $43.30 \%$ & $38.03 \%$ & $38.43 \%$ \\
\hline & Direxion Daily Energy Bull 3× Shares & $66.36 \%$ & $64.01 \%$ & $76.84 \%$ \\
\hline & Direxion Daily S \& P 500 Bull 3× Shares & $43.64 \%$ & $38.10 \%$ & $38.34 \%$ \\
\hline & ProShares Ultra Real Estate & $31.08 \%$ & $26.10 \%$ & $29.00 \%$ \\
\hline & Direxion Daily Technology Bull 3× Sahres & $50.06 \%$ & $39.89 \%$ & $44.24 \%$ \\
\hline \multirow{10}{*}{ 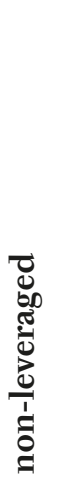 } & SPDR S \& P 500 ETF Trust & $14.13 \%$ & $12.31 \%$ & $12.54 \%$ \\
\hline & iShares CoreS \& P 500 ETF & $14.13 \%$ & $12.39 \%$ & $12.52 \%$ \\
\hline & Vanguard Total Stock Market Index & $14.59 \%$ & $12.58 \%$ & $12.54 \%$ \\
\hline & Vanguard Value Index Fund & $13.90 \%$ & $12.26 \%$ & $12.19 \%$ \\
\hline & iShares Core S \& P Small Cap ETF & $17.12 \%$ & $14.32 \%$ & $13.57 \%$ \\
\hline & Vanguard Total Bond Market Index & $3.30 \%$ & $3.26 \%$ & $356.20 \%$ \\
\hline & Energy Select Sector SPDR Fund & $22.44 \%$ & $21.00 \%$ & $24.64 \%$ \\
\hline & iShares U.S. Financials ETF & $16.68 \%$ & $13.07 \%$ & $12.92 \%$ \\
\hline & SPDR S \& P BIOTECH ETF & $27.71 \%$ & $31.66 \%$ & $33.72 \%$ \\
\hline & Vanguard REIT Index Fund & $16.35 \%$ & $14.04 \%$ & $15.88 \%$ \\
\hline
\end{tabular}


Appendix 2: Systematic risk of leveraged and non-leveraged ETF's

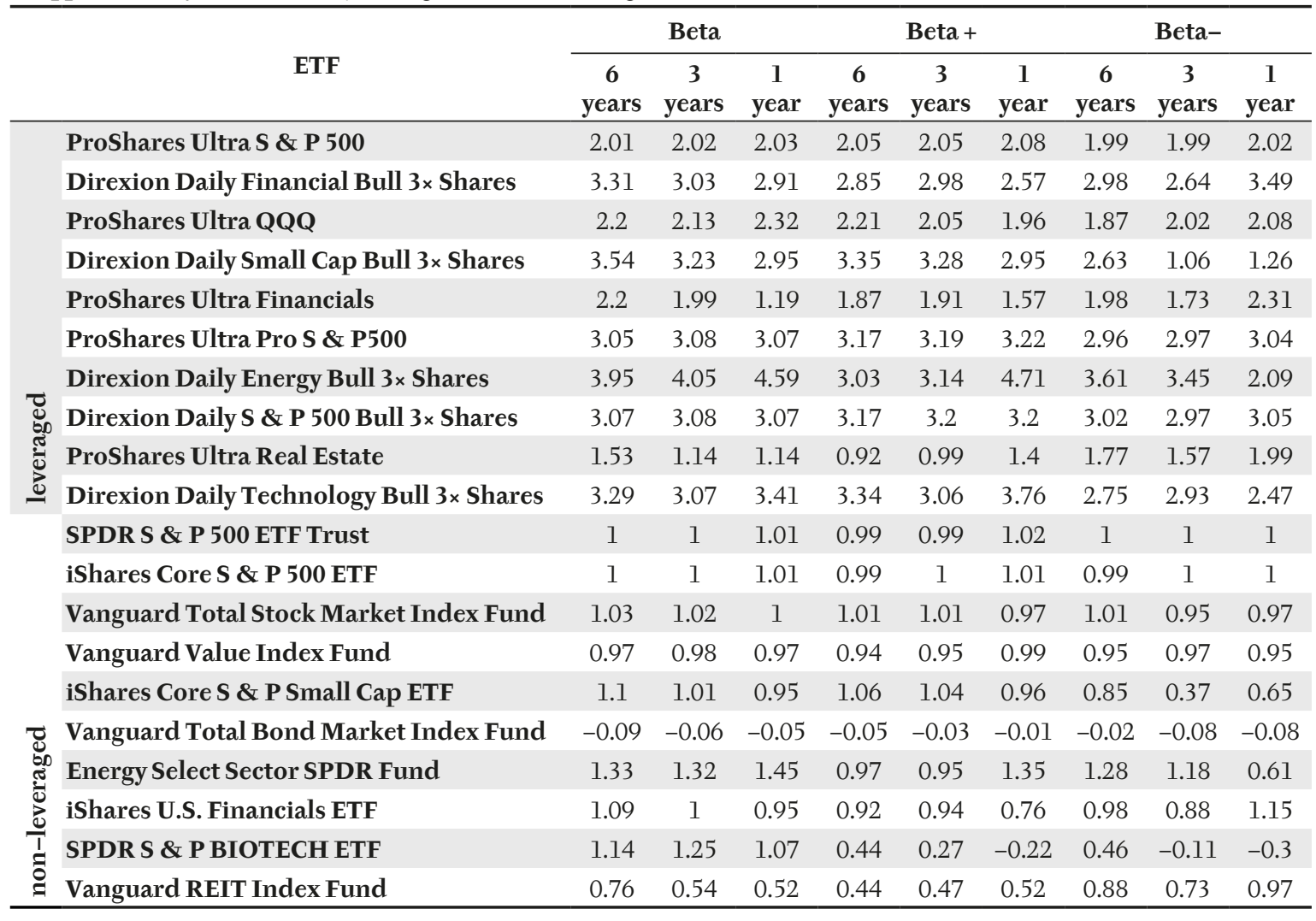

